\title{
Electronic Structure of MoS2 and WS2 Nanotubes
}

\author{
1, Rafael de Alencar Rocha (PG), ${ }^{2}$ Renato Batista Santos (PQ) and ${ }^{3}$ Luiz \\ Antônio Ribeiro Júnior ( $P Q)$ \\ ${ }^{1}$ Institute of Physics, University of Brasília, Brasília, 70910-970, DF, Brazil, ${ }^{2}$ Federal Institute for \\ Education, Science, and Technology of Bahia, Senhor do Bonfim, Bahia, 48.970-000, Brazil, \\ ${ }^{2}$ International Center for Condensed Matter Physics, University of Brasília, P.O. Box 04531, \\ 70.919-970, Brasília, DF, Brazil \\ Keywords: Transition Metal Dichalcogenides, Nanotubes, MoS2, WS2, Electronic Structure.
}

\section{Introduction}

The layered transition-metal dichalcogenides (TMDs) MoS2 and WS2 show remarkable electronic properties that are interesting for develop a new class of optoelectronic devices [1]. Interestingly, some external effects can modify the direct their band gap into an indirect one by inducing, therefore, a semiconductor-metal transition [2]. The layered TMDs have been extensively studied in the past few years and a deep understanding about their important optoelectronic properties have being obtained [2]. However, the same kind of attention has not been paid when it comes to single-walled nanotubes formed by TDMs. Here, we investigate the electronic structure of armchair and zigzag single-walled MoS2 and WS2 nanotubes using Density Functional Theory. The present study may provide guidance for a better understanding of the essential TMD features by making a connection between electronic and geometrical properties of these materials.

\section{Methodology}

The DFT calculations presented here were carried out using a commercial software package named Materials Studio, which is developed by Accelrys Inc. This software includes the modules DMol3 and CASTEP, that use DFT with a numerical radial function basis and with a_plane wave basis set, respectively, to calculate the electronic properties of molecules, clusters, surfaces and crystalline solid materials from first principles. Here we calculate the electronic and optical properties of MoS2 and WS2 nanotubes by using the LDA/PW91 and GGA/PBE DFT methods, as implemented in the modules CASTEP and DMol3.

\section{Results}

The results of our DFT calculations show that TMD nanotubes present similar band gaps for all the methods. The considered structures are $(6,6)$ for armchair and $(10,0)$ for zigzag nanotubes. Moreover, from our results it is possible to realize the semiconducting-like trend for these structures. The figure 
below show the nanotube configuration as well as the band gap for these structures. We can see that the variation direct and and indirect band gaps for the tubes 10,10 and 6,6, respectively.

(a)

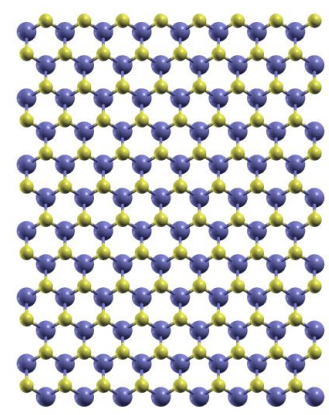

(b)
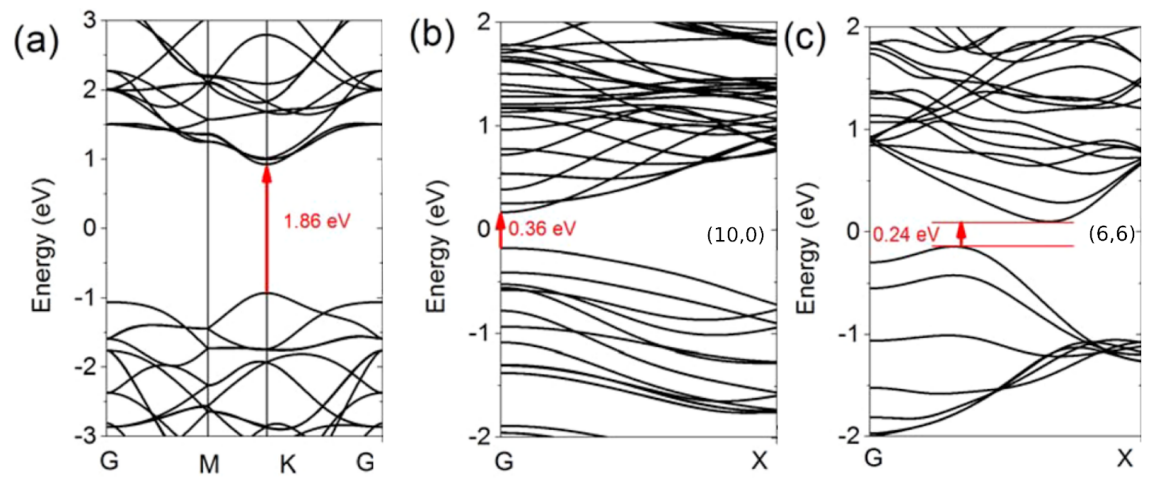

\section{Conclusions}

In summary, the method adopted to investigate the properties electronic of the structure MoS2 and WS2 through the DFT calculation show the reduced band gap in comparison with the sheet, variation of band gap for nanotubes $(10,0)$ and $(6,6)$.

\section{Acknowledgements}

The authors gratefully acknowledge the financial support from the Brazilian Research Councils CAPES and FAPDF. L.A.R.J. gratefully acknowledges the financial support from the Brazilian Research Council FAPDF grant 0193.000942/2015 and 193.001.511/2017. L.A.R.J. also wishes to thank the Brazilian Ministry of Planning, Budget and Management (Grant DIPLA 005/2016).

\section{References}

[1] Kai-Xuan Chen, Xiao-Ming, Dong-Chuan. Thermoelectric Properties of Transition Metal Dichalcogenides: From Monolayers to Nanotubes

[2] Jin Xiao, Mengqiu Long, Xinmei Li. Theoretcal Prediction of Electronic Structure and Carrier Mobility in Single-walled MoS2 Nanotubes 